\title{
Nuclear data research at the Los Alamos Neutron Science Center
}

\author{
R.C. Haight ${ }^{\mathrm{a}}$ \\ Los Alamos Neutron Science Center, Los Alamos, NM 87545, USA
}

\begin{abstract}
The Los Alamos Neutron Science Center is based on an $800-\mathrm{MeV}$ high current proton linear accelerator, which is used to produce intense pulses of neutrons over 16 orders of magnitude in energy from ultra-cold neutrons to neutrons with energies up to $800 \mathrm{MeV}$. The four separate neutron production areas and the neutron energy ranges at each are: (1) the Weapons Neutron Research facility $(0.1$ to $800 \mathrm{MeV})$; (2) the Lujan Center with moderated neutrons (cold - $500 \mathrm{keV}$ ); (3) the Lead Slowing-Down Spectrometer $(0.1 \mathrm{eV}$ to $200 \mathrm{keV})$; and (4) the Ultracold Neutron Research facility (ultracold). Because of the flexibility of the accelerator, a wide range of neutron source intensities, pulse widths, and intensities is possible. Nuclear data measurements are conducted with the first three of these sources. Present research activities include experiments (with the instruments used) on high-resolution gamma-ray production by neutrons (GEANIE), neutron capture (DANCE), neutron and gamma-ray emission from fission and other reactions (FIGARO), charged-particle emission (NZ), neutron-induced fission (FISSION), and fission on small samples with a Lead Slowing Down Spectrometer (LSDS). With these capabilities, new approaches to studying neutron-induced reactions are yielding information on a wide range of nuclear data: gamma-ray emission including multiplicity and energy distributions, capture-to-fission ratios, transmutation reactions, emission probabilities for charged particles and neutrons, and cross sections on nuclides off the valley of stability. For some of the instruments, reactions can be studied on very small or short-lived samples. Analysis of the accuracy and precision of experiments is now being carried out in cooperation with data evaluators in order to improve the files of data covariances. Furthermore, the data are providing stringent tests of nuclear reaction models. Researchers from other laboratories in the US and from other countries collaborate in or lead many of these studies. The overview, presented here, of recent and on-going nuclear data research highlights several of the unique research opportunities made possible by the LANSCE neutron sources and specialized instrumentation. Possibilities for future advances are outlined.
\end{abstract}

\section{Introduction}

The Los Alamos Neutron Science Center (LANSCE) is a versatile user facility for research based on a high current linear accelerator that produces a variably pulsed proton beam at $800 \mathrm{MeV}$ as well as at lower energies [1]. The time structure of the beam can be optimized by a proton-storage ring (PSR). Principal uses for this facility are for neutron nuclear science, neutron scattering, proton radiography and isotope production. This paper focuses on the activities at LANSCE for neutron nuclear data. Because of time and space limitations at this Conference, many details will not be covered and the reader is referred to refs. [2-4].

With this powerful neutron source facility, new instruments are needed to utilize the new capabilities. Ideally they have improved efficiency and specificity in addition to handling high count rates. We have developed several of these instruments for studying fission and the production of gammarays, neutrons, and charged particles by neutrons. Updates on the status of these instrument and a few illustrative examples of the research done with them are presented here.

In the design of experiments, we are also users of nuclear data. Some examples will be given where nuclear data and radiation transport codes have played key roles in designing experiments at LANSCE.

\footnotetext{
${ }^{a}$ Presenting author, e-mail: haight@lanl.gov
}

\section{Neutron Sources at LANSCE}

Neutrons are produced at LANSCE by spallation reactions of 800-MeV protons on tungsten targets. Four experimental areas are used and are referred to as the Weapons Neutron Research facility (WNR), the Manuel Lujan Jr. Neutron Scattering Center (Lujan Center), Ultra-cold neutron (UCN) facility, and the Lead Slowing-Down Spectrometer (LSDS). These sources produce neutrons over 16 orders of magnitude in energy. They differ by the amount of beam that they can take, by the pulse width of the beam, and whether or not moderators are used to degrade the energy of the neutrons. The present beam capabilities of each area are given in table 2.1. The layout of the facility is presented in ref. [1].

\subsection{WNR}

The WNR neutron source uses the linac beam incident on a water-cooled tungsten cylinder $7 \mathrm{~cm}$ long and $3 \mathrm{~cm}$ in diameter to produce fast neutrons in a usable range from $\sim 0.1$ to $600 \mathrm{MeV}$. The beam consists of $200 \mathrm{ps}$ bunches of about $8 \times$ $10^{8}$ protons per bunch spaced according to the experimental requirements, with a typical spacing of $1.8 \mu \mathrm{s}$. Six flight paths from 9 to 90 meters in length view the neutron source. The production angles of 15 to 90 degrees allow the emphasis (at 15 degrees) or de-emphasis (90 degrees) of the high energy part of the source spectrum. Furthermore, it is customary to add attenuators such as $\mathrm{CH}_{2}, \mathrm{~Pb}$, or other materials to shape the neutron spectrum at the experimental station. 
Table 1. Neutron sources at LANSCE. The beam current is given as time averages.

\begin{tabular}{lccc}
\hline Facility & $\begin{array}{c}\text { Beam current } \\
(\mu \text { A-time avg) }\end{array}$ & $\begin{array}{c}\text { Pulses } \\
\text { per sec }\end{array}$ & $\begin{array}{c}\text { Beam } \\
\text { pulse width } \\
(\mathrm{ns})\end{array}$ \\
\hline WNR & $1.7^{*}$ & $13,890^{*}$ & $0.2 \mathrm{~ns}$ \\
Lujan & 100. & 20 & $135 \mathrm{~ns}$ \\
LSDS & 1. & 40 & $100 \mathrm{~ns}$ \\
UCN & 2. & 0.1 & long \\
\hline
\end{tabular}

* WNR is presently limited to these values by aging components in the accelerator. These components are planned to be replaced for a factor of 2.5 improvement in the beam current and the number of pulses per second.

\subsection{Lujan center}

The Lujan neutron source takes the beam from the Proton Storage Ring (PSR), which compresses a 625 microsecondlong macropulse into an intense pulse of $5 \mu \mathrm{C}$ that is directed to a complex target-moderator system [5]. The typical rate of these compressed pulses is 20 pulses per second. Beam lines look at neutrons from these moderators, which, for the nuclear data activities, are water moderators. Flight paths of 7 to 60 meters are possible. The usable neutron energy range for nuclear data experiments is from thermal to about $200 \mathrm{keV}$.

\subsection{Lead Slowing-Down Spectrometer}

The Lead Slowing-Down Spectrometer (LSDS) $[6,7]$ is described in section 3.5 below. It is driven by the linac beam for low-intensity operation, with a beam envelope on the order of $100 \mathrm{~ns}$. For production runs where a larger number of protons is required in $100 \mathrm{~ns}$, the linac beam is compressed by the PSR, which can run at 40 pulses per second. The beam is incident on an air-cooled tungsten cylinder, $25-\mathrm{cm}$ long and $7 \mathrm{~cm}$ in diameter. Useful neutron energies are from $0.1 \mathrm{eV}$ to $100 \mathrm{keV}$.

\subsection{Ultra-cold neutrons}

The facility for ultra-cold neutrons (UCN) $[8,9]$ has been developed recently for basic physics studies of the neutron and its decay properties. Ultra-cold neutrons have a velocity of less than $7 \mathrm{~m} / \mathrm{s}$, which corresponds to an energy of less than 250 nano-electron volts. At LANSCE, the linac beam is incident on a $10 \mathrm{~cm}$-long tungsten target and the spallation neutrons are moderated to ultra-cold energies by an assembly of beryllium, graphite, $\mathrm{CH}_{2}$, liquid helium and solid deuterium. The beam is on for up to $100 \mathrm{~ms}$ and then turned off so that the radiation background during the UCN measurements, which take many seconds, is extremely low. The repetition rate of the beam is on the order of 0.01 pulses per second.

\section{Instruments for research}

To take advantage of the capabilities of the LANSCE neutron sources, several specialized instruments have been developed over the past few years (see fig. 1). The schedule

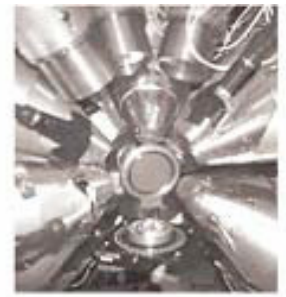

\section{GEANIE}

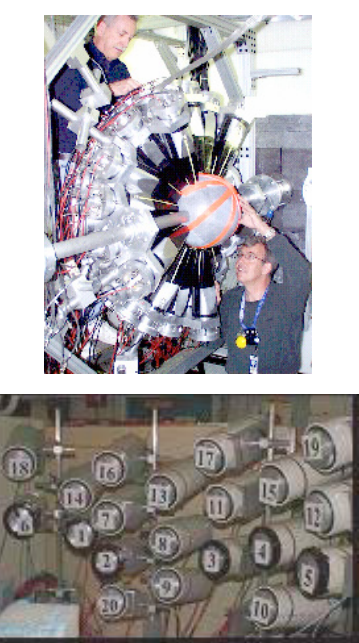

\section{DANCE}

FIGARO

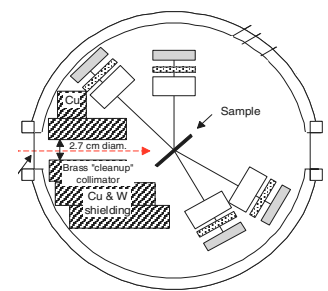

NZ

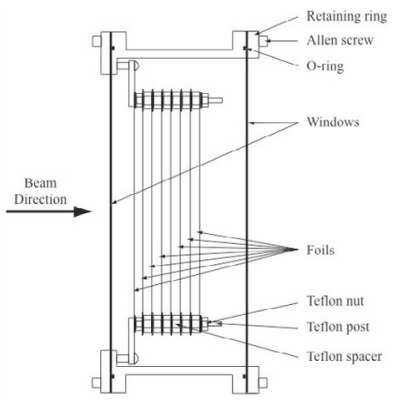

Fission

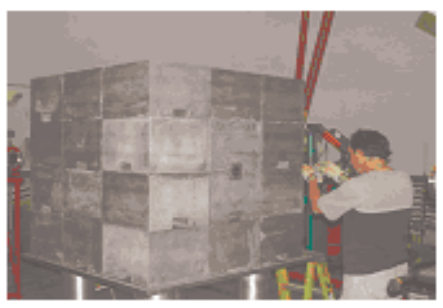

LSDS

Fig. 1. Instruments used at LANSCE for research on neutron nuclear data.

of investments in these instruments has been guided by the needs for certain types of nuclear data for our Laboratory's programs. A common theme of most of these instruments is that they are arrays of detectors, e.g., for gamma-rays, neutrons, and charged particles. These arrays allow efficient use of the beam and they also make possible new types of 
measurements such as coincidences and overall multiplicity of the emitted radiations. All but one of the instruments described here, as well as others provided by outside users, work on a time-of-flight principle in that they are located 6 to 90 meters from the neutron source and the incident neutron energy is determined by its flight time from the source to the sample. The LSDS described below effectively works on the same time-to-energy correlation.

\subsection{GEANIE}

The Germanium Array for Neutron-Induced Excitations (GEANIE) is an array of up to 26 high-purity germanium gamma-ray detectors and is described in this Conference by R. Nelson [10]. Nuclear structure and nuclear reaction cross sections induced by neutrons from $100 \mathrm{keV}$ to $200 \mathrm{MeV}$ are studied with this instrument.

The level structure of nuclei and some nuclear lifetime information is obtained by the high-resolution measurement of gamma-rays emitted after the nucleus is excited by inelastic scattering or by a nuclear reaction. The yield of these gamma rays as a function of incident neutron energy gives information on the excitation energy of a level excited by inelastic scattering or on the specific nucleus produced in the reaction. An example of the power of this technique is in substantial increase in the knowledge of the levels of ${ }^{197} \mathrm{Au}$ [11] where 52 new transitions and 32 new levels were found.

Nuclear reaction cross sections, such as $(n, 2 n)$, are determined by the yield of the gamma rays in the residual nucleus. If the excited levels of the residual nucleus decay by cascades through one or just a few low-lying states, the cross section can be determined rather reliably. A nuclear model code is used to correct for the unobserved transitions and to relate the measured yields to the reaction cross section. This approach is described in detail, for example in ref. [12].

The GEANIE array already is unique in its use of planar HPGe detectors to get better timing and to go down to gammaray energies of $20 \mathrm{keV}$. Future improvements in the array will include more efficient detectors, such as so-called called "clover" HPGe detectors to improve the efficiency for gammagamma coincidence measurements, which in turn will allow better specificity for identifying the residual nuclide especially for odd-A or odd-odd nuclides, more sensitive detection of weaker gamma-ray yields, and, for fission, better efficiency in measuring gamma rays and X-rays from correlated fission products.

\subsection{DANCE}

The Detector for Advanced Neutron Capture Experiments (DANCE) is a highly segmented array of $160 \mathrm{BaF}_{2}$ crystals arranged as an approximately $4 \pi$-calorimeter for neutron capture experiments including measurements of cross sections, gamma-ray multiplicities and capture-to-fission ratios. DANCE is located on a 20-meter beam line at the Lujan Center and uses neutrons from thermal to $200 \mathrm{keV}$. The high efficiency of the $4 \pi$-calorimeter coupled with the intense

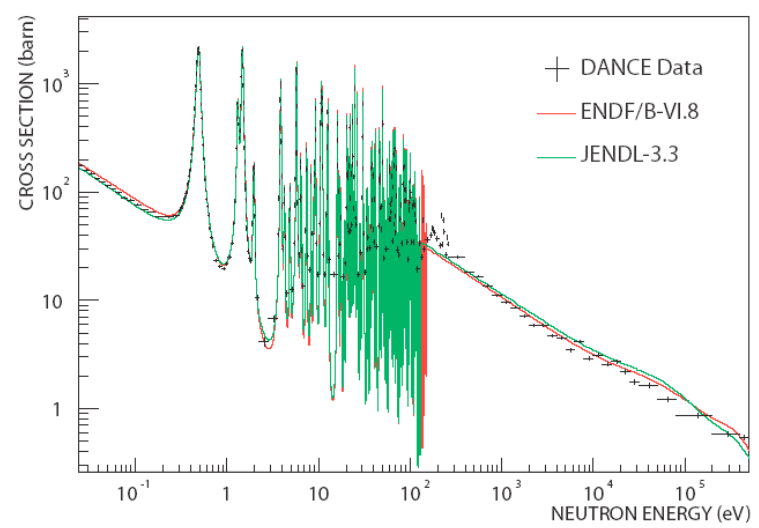

Fig. 2. Neutron capture cross section on ${ }^{237} \mathrm{~Np}$ measured at DANCE with a sample of $0.44 \mathrm{mg}$.

neutron beam allows measurements with milligram or even sub-milligram samples.

The efficiency of DANCE is approximately $95 \%$ for detection of a single gamma ray at $1 \mathrm{MeV}$. A neutron capture event usually produces several gamma rays, the pulse heights of which are summed in this calorimeter. If all of the capture energy is converted into gamma rays and into scintillation light, then ideally the summed pulse heights correspond to $\Sigma \mathrm{E}_{\gamma}=\mathrm{Q}+\mathrm{E}_{\mathrm{n}}$, where $\mathrm{Q}$ is the Q-value for neutron capture and $E_{n}$ is the incident neutron energy corrected for the centerof-mass motion. In reality, some of the energy is lost and therefore the peak is broadened toward lower pulse heights. Summing the peak over an energy range down to half the peak still yields an efficiency for detecting capture of more than $70 \%$ when we consider also the effect of a ${ }^{6} \mathrm{LiH}$ spherical shell placed between the sample and the scintillators to absorb scattered neutrons.

Because of the high segmentation of the array, the multiplicity of gamma rays can be well determined from the number of detectors fired [13]. For fission, the multiplicity of gamma rays is generally higher than that for neutron capture. Furthermore, for fission there is a distribution of the total energy emitted as gamma rays from the fission fragments. By placing windows on the energy and the multiplicity of gamma rays, the capture-to-fission ratio can be obtained. Bredeweg et al. describe this method in this conference [14].

A recent example of DANCE data cross section data is given in figure 2 for ${ }^{237} \mathrm{~Np}(\mathrm{n}, \gamma)$ [15]. Other results from this instrument are presented at this Conference [14, 16, 17].

Upgrades to DANCE could include faster data processing and the inclusion of one or more HPGe detectors in the array. At present we record the signals from each $\mathrm{BaF}_{2}$ detector with two $500 \mathrm{MSamples} / \mathrm{s}, 8$-bit waveform digitizers. The 320 channels of data are processed by on-board computers in each of the 14 PCI crates to give pulse height, pulse shape and time information, and then routed to a central computer for recording and display. The Lujan beam is pulsed at 20 pulses per second and so the data acquisition needs to be completed in less than 50 milliseconds. For high event rates, such as with larger samples, this data stream stresses the capabilities of the onboard computers and data transfer, a problem that can be 
solved with newly-available computers. Addition of one or more HPGe detectors would allow further selectivity of the events to those for neutron capture on the target sample. These high-resolution detectors would also open up studies of the details of the gamma-ray cascade following neutron capture and the determination of the spins and parities of the capture states.

\subsection{FIGARO}

The Fast neutron-Induced Gamma-Ray Observer (FIGARO) consists of an array of 20 liquid scintillation detectors, with pulse-shape discrimination between neutrons and gamma rays, and two or three gamma-ray detectors with the goal of studying neutron emission spectra and multiplicities and gammaray production. The detector arrays view a sample located 22.74 meters from the WNR neutron source. Double timeof-flight is measured to identify the energy of the incident neutron and the energy of the emitted neutron(s) and also to identify gamma-rays over flight paths of $\sim 1$ meter from the sample. The time of arrival of the source neutron at the sample is determined by the gamma-ray(s) emitted in the reaction, or, for fission studies, by placing the fissionable material in a fission chamber in the beam.

Fission neutron spectra and multiplicities have been measured with FIGARO for ${ }^{235} \mathrm{U}$ and ${ }^{238} \mathrm{U}$ for incident neutron energies from $1 \mathrm{MeV}$ to $200 \mathrm{MeV}$ [18]. Measurements on ${ }^{237} \mathrm{~Np}$ are reported at this meeting [19].

Another use of FIGARO is to measure neutron emission spectra from inelastic scattering and other reactions. Here the time marker for the reaction is determined by a gamma-ray detector. For many even-even nuclides, the decay of most of the excited states proceeds by a cascade that goes through the lowest $2+\rightarrow$ ground state transition. The gamma ray emitted by this transition is often in the energy range easily detected by a gamma-ray detector. An example is for neutron emission spectra from neutrons on natural where $2+\rightarrow$ g.s. gamma rays for the two major isotopes, ${ }^{58,60} \mathrm{Ni}$, were unresolved in the experiment [20].

An upgrade to FIGARO is expected soon in the form of a lanthanum-halide scintillator. Up to now, we have used $\mathrm{BaF}_{2}$ and $\mathrm{BGO}$ scintillators and a planar HPGe detector for the gamma ray. The former two have sufficiently good timing ( $\sim 1 \mathrm{~ns}$ ) but modest resolution, whereas the HPGe detector has excellent resolution but only $\sim 5 \mathrm{~ns}$ timing. The lanthanum halide scintillator has excellent timing characteristics and resolution that is about 4 times better than the $\mathrm{BaF}_{2}$ scintillator so that, for example, the gamma-ray lines in ${ }^{58,60} \mathrm{Ni}$ at 1.454 and $1.332 \mathrm{MeV}$ respectively will be easily resolved.

\section{$3.4 \mathrm{NZ}$}

Charged particles produced by neutron-induced reactions are studied by the NZ instrument, which consists of 4 detector telescopes for identifying the particle type and measuring its energy. The telescopes consist of a low pressure (20 torr) proportional counter, a silicon surface barrier detector, and a $\mathrm{CsI}(\mathrm{Tl})$ scintillator and they view the thin-foil sample which is located $15.07 \mathrm{~m}$ from the neutron source. Experiments at this instrument include standards measurements of the ${ }^{6} \mathrm{Li}(\mathrm{n}, \mathrm{t})^{4} \mathrm{He}$ cross section [21], production of hydrogen and helium in structural materials for advanced reactors [22], and level density studies [23].

\subsection{Fission}

Fission cross sections are measured both at the Lujan Center and at WNR over 10 orders of magnitude in incident neutron energy, thermal to $200 \mathrm{MeV}$. At present, a multifoil fission chamber [24] is used to measure the fission cross section ratios. Data for ${ }^{237} \mathrm{~Np}(\mathrm{n}, \mathrm{f})$ have been recently reported [25]. These measurements are part of an extensive program to improve the fission cross section data for actinides of importance to the development of advanced reactors.

Future developments in fission experiments are being developed and include time-projection chambers and dual-arm spectrometers.

\subsection{Lead slowing-down spectrometer}

To study the fission of rare actinides or reactions with very small cross sections, a high intensity neutron source is required. Together with colleagues from the CEA and RPI, we are developing a 1.2 meter-cube Lead Slowing-Down Spectrometer (LSDS) driven by pulsed protons from the PSR $[7,26]$. This work is described is another contribution to this conference [27]. To date we have used up to a 1 microampere (time-averaged) beam pulsed 40 times per second with a pulse width of $100 \mathrm{~ns}$. Fission detectors are placed in channels in the lead and the fission events are recorded as a function of time after the beam pulse. The time-energy relationship for an LSDS is

$$
\left\langle E_{n}\right\rangle=K /\left(t+t_{0}\right)^{2}
$$

where $\left\langle E_{n}\right\rangle$ is the mean neutron energy and $K$ and $t_{0}$ are constants characteristic of the spectrometer. The large increase in neutron flux in the LSDS is indicated in figure 3. The penalty one pays for the very large flux is a decrease in resolution, which is at best about $30 \%$ in $\triangle \mathrm{E} / \mathrm{E}$.

With this spectrometer we have demonstrated a fission cross section measurement on ${ }^{239} \mathrm{Pu}$ on a sample of 9.87 nanograms [26].

Future development of the LSDS will include remotely insertable detectors, such as solar cells that now deteriorate in about a day of running, and new types of ion chambers [28].

\section{Covariance data}

An important new direction for experimentalists at LANSCE is the discussion of the covariances in the uncertainties of our results. This activity involves discussions in depth with data evaluators in order to specify the covariances that are being entered into the evaluated data libraries such as ENDF/BVII. The uncertainties including those of sample composition, impurities, dead time, neutron fluence normalization all need 


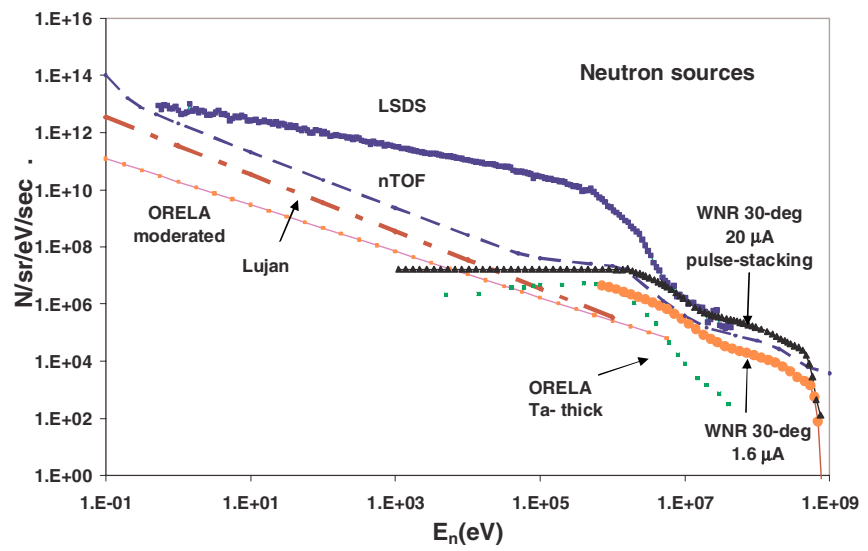

Fig. 3. Neutron source strengths at WNR, Lujan and LSDS at LANSCE. The source strengths for $n_{-}$TOF [32] and ORELA [33] are also shown. The values for the LSDS are for an equivalent flight path of 6 meters, which has the same time-energy relationship although the resolution of the LSDS is no better than $30 \%$ in $\triangle \mathrm{E} / \mathrm{E}$. With pulse stacking, the WNR source strength can be significantly increased and the range extended down from $1 \mathrm{MeV}$ to about $1 \mathrm{keV}$.

to go into the final covariance data files. An example of these covariances is given for ${ }^{237} \mathrm{~Np}(\mathrm{n}, \mathrm{f})$ in ref. [29]. This subject is discussed elsewhere in this conference.

\section{Uses of nuclear data}

In addition to providing nuclear data, the LANSCE program of research also uses nuclear data for the design of experiment and for radiation shielding calculations. Here are two examples.

\subsection{LSDS shielding}

The LSDS is located in a room that is not usually used for high-radiation experiments and therefore has only moderate shielding. For radiation protection, we needed to install three panels of shielding in the entryway maze and the question was whether the shielding would be sufficient. The MCNPX calculations [30] involved a complex three-dimensional simulation of the room, the entryway and the additional shielding. To give confidence that the calculational results could be believed, two previous configurations were calculated and the results were consistent with measurements. The room and the hallway were the same for these configurations as for the LSDS, but the neutron source, moderated by 20 tons of lead, and the additional shielding were quite different. For the LSDS experiments, careful measurements were made at gradually increasing beam levels in the areas where personnel had access. The measurements agreed to within a factor of 2 of the calculated values and no additional shielding was required.

\subsection{Ultra-cold neutron facility}

As mentioned earlier, the UCN facility for producing ultracold neutrons (UCN) is based on neutron production in a tungsten target and moderation by a large assembly of graphite, beryllium, structural materials (mostly aluminium), liquid helium (LHe) and solid deuterium (SD). The crucial aspect of this design is to keep the heating of the cryogenic materials to a minimum, for an increase in temperature from $5 \mathrm{~K}$ to $10 \mathrm{~K}$ of the SD reduces the production of UCN by a large factor. This UCN source uses a proton beam pulsed only every 10 seconds, but the heating is due to the neutrons that are incident first on the LHe and SD and thus must be calculated correctly. The design was based on numerous simulations $[8,31]$ and the results were well verified by measurements.

\section{Improvements in neutron production}

The neutron source strengths for the WNR, Lujan and LSDS facilities are shown in figure 3 and compared with the performances of $n_{-}$TOF [32] and ORELA [33]. Whereas WNR has outstanding characteristics in the high energy range and Lujan at the low energies, the range between about $1 \mathrm{keV}$ and a few $\mathrm{MeV}$ needs improvement at LANSCE. This range is central to the development of fast reactors, it is key to understanding nucleosynthesis in stars, and there are other applications.

To improve the LANSCE performance in this range, the concept of "pulse stacking" has been proposed [34,35]. Short pulses from the linac would be accumulated on top of each other in the PSR and then switched to the WNR target. In this way, the beam current could be retained, or even improved, and the pulse spacing could be chosen to best suit the experiment. The increase in neutron source strength can be as much as a factor of 30 at certain energies in this $1 \mathrm{keV}-$ $5 \mathrm{MeV}$ range.

\section{Summary}

LANSCE is a versatile, multipurpose facility for the production of neutron nuclear data. The neutron sources are intense over 16 orders of magnitude in neutron energy. Instruments, many of them arrays of detectors, are used to measure cross sections and to deduce nuclear structure by the detection of emitted gamma rays, neutrons, and charged particles or of fission events. The facility is already very productive and promises to be more so with planned improvements in the detectors and the neutron sources.

The author would like to thank his colleagues and the many other users of LANSCE for their accomplishments that are outlined here. This work has benefited from the use of the Los Alamos Neutron Science Center at the Los Alamos National Laboratory. This facility is funded by the US Department of Energy and operated by Los Alamos National Security, LLC under contract DE-AC52 06NA25396.

\section{References}

1. P.W. Lisowski, K.F. Schoenberg, Nucl. Instrum. Meth. Phys. Res. A 562, 910 (2006).

2. P.W. Lisowski, C.D. Bowman, G.J. Russell, S.A. Wender, Nucl. Sci. Eng. 106, 208 (1990). 
3. A. Michaudon et al. (to be published)

4. WNR web site: http://wnr.lanl.gov

5. T. Inoa et al., Nucl. Instrum. Meth. Phys. Res. A 525, 496 (2004).

6. T. Granier et al., Nucl. Instrum. Meth. Phys. Res. A 506, 149 (2003).

7. D. Rochman et al., Nucl. Instrum. Meth. Phys. Res. A 550, 397 (2005).

8. R.E. Hill et al., Nucl. Instrum. Meth. Phys. Res. A 440, 674 (2000).

9. A. Saunders et al., Phys Lett. B 593, 55 (2004).

10. R.O. Nelson et al. (these proceedings).

11. N. Fotiades et al., Phys. Rev. C 71, 064314 (2005).

12. L.A. Bernstein et al., Phys. Rev. C 65, 021601 (2001).

13. M. Heil et al., Nucl. Instrum. Meth. Phys. Res. A 459, 229 (2001).

14. T.A. Bredeweg et al. (these proceedings).

15. E.-I. Esch et al. (submitted to Phys. Rev. C) (2007).

16. D. Vieira et al. (these proceedings).

17. W. Parker et al. (these proceedings).

18. T. Ethvignot et al., Phys. Rev. Lett. 94, 052701 (2005).

19. J. Taieb et al. (these proceedings).

20. D. Rochman et al., Nucl. Instrum. Meth. Phys. Res. A 523, 102 (2004).
21. M. Devlin et al. (these proceedings).

22. R.C. Haight (these proceedings).

23. S.M. Grimes, T.N. Massey, C. Brune (2007) (private communication).

24. S.A. Wender et al., Nucl. Instrum. Meth. Phys. Res. A 336, 226 (1993).

25. F. Tovesson, T.S. Hill, Phys. Rev. C 75, 034610 (2007).

26. D. Rochman et al., Nucl. Instrum. Meth. Phys. Res. A 564, 400 (2006).

27. S.A. Wender et al. (these proceedings).

28. C. Romano et al., Nucl. Instrum. Meth. Phys. Res. A 562, 771 (2006).

29. F. Tovesson, T.S. Hill (submitted to Phys. Rev. C) (2007).

30. C. Kelsey (2005) (private communication).

31. A. Saunders (2007) (private communication).

32. The $\mathrm{n}_{-} \mathrm{TOF}$ collaboration, CERN/SPSC 99-8, SPSC/P 310 (1999).

33. K.H. Boeckhoff, A.D. Carlson, O.A. Wasson, J.A. Harvey, Nucl. Sci. Eng. 106, 192 (1990).

34. A. Michaudon, S.A. Wender, Performance of the LANSCE-WNR facility as an intense pulsed neutron source for neutron nuclear physics, LA-UR-90-4355, pp. 45-46 (1990).

35. S.A. Wender (2007) (private communication). 